\title{
Mejores prácticas para el lavado y manipulación de la bolsa de alimentación enteral Kangaroo ${ }^{1}$
}

Institución: Hospital San Rafael de Alajuela

Consuelo Cubero-Alpízar ${ }^{2}$ Luz Marina Flores Martínez ${ }^{3}$ Jenny Rodríguez Rodríguez ${ }^{4}$

\section{COMO CITAR}

Cubero-Alpízar, C., Flores, L.M. y Rodríguez, J. (2016). Mejores prácticas para el lavado y manipulación de la bolsa de alimentación enteral Kangaroo. Rev. Enfermería Actual en Costa Rica, 31, 1-10. DOI: http://dx.doi.org/10.15517/revenf.v0i31.25386

\section{RESUMEN}

Introducción: La nutrición enteral es una valiosa herramienta en la terapia de los pacientes hospitalizados, especialmente en las unidades de cuidado intensivo y en pacientes que, por sus patologías, pierden su habilidad de ingerir alimentos.

Metodología: se aplicó la metodología que señala la práctica clínica basada en evidencia (PCBE) que trata de localizar hallazgos que respondan a la pregunta clínica planteada mediante el estudio y análisis de investigaciones primarias y originales de alta calidad científica.

Resultados: Ningún estudio respondió directamente a la pregunta, sin embargo, varias investigaciones aluden que el lavado de manos y el uso de guantes limpios funcionan como estrategia para la manipulación y lavado de este dispositivo. La calidad de los estudios encontrados es baja ya que provienen de investigaciones basadas en la opinión de expertos y revisión de literatura de poca calidad.

Conclusiones. No se encuentra evidencia significativa que respalde el uso de técnica aséptica médica y quirúrgica para el manejo y lavado de la bolsa Kangaroo de alimentación enteral.

Palabras clave: lavado-de-bolsa-kangaroo, nutrición-enteral, práctica-clínica.

\footnotetext{
${ }^{1}$ Fecha de recepción: 26 de octubre del 2015

Fecha de aceptación: 15 de febrero del 2016

${ }^{2}$ Enfermera. Docente de la Escuela de Enfermería. Universidad de Costa Rica. Costa Rica. Correo electrónico: ccuberoalpzar@gmail.com

${ }^{3}$ Enfermera. Hospital San Rafael de Alajuela. Caja Costarricense del Seguro Social. Costa Rica. Correo electrónico: luzf7494@gmail.com

${ }^{4}$ Enfermera. Hospital San Rafael de Alajuela. Caja Costarricense del Seguro Social. Costa Rica. Correo electrónico: jeyronurse04@hotmail.com
} 


\title{
Best practices for washing and handling of the bag Kangaroo enteral feeding ${ }^{1}$
}

Institution: San Rafael de Alajuela Hospital

Consuelo Cubero Alpízar ${ }^{2}$

Luz Marina Flores Martínez ${ }^{3}$

Jenny Rodríguez Rodríguez ${ }^{4}$

\section{CITED}

Cubero-Alpízar, C., Flores, L.M. y Rodríguez, J. (2016). Best practices for washing and handling of the bag Kangaroo enteral feeding. Rev. Enfermería Actual en Costa Rica, 31, 1-10. DOI: http://dx.doi.org/10.15517/revenf.v0i31.25386

\begin{abstract}
Introduction. Enteral nutrition is a valuable tool in the therapy of hospitalized, especially in intensive care units, and patients for their diseases lose their ability to ingest food patients.

Method. The methodology pointing evidence-based clinical practice (PCBE) dealing with the study and analysis of primary and original research of high scientific quality, locating findings that respond to the clinical question posed was applied.
\end{abstract}

Results. No study directly answer the question, however, several investigations allude to the fact that washing hands and using clean gloves as a strategy for handling and washing of this device. The quality of the studies found is low because they come from research based on expert opinion and literature review of low quality.

Conclusion. No significant evidence to support the use of medical and surgical aseptic technique for handling and washing Kangaroo Enteral feed bag was found.

Key words: clinical-practice, enteral-nutrition, wash-bag

\footnotetext{
${ }^{1}$ Date of receipt: october 26,2015

Date of acceptance: february 15, 2016

${ }^{2}$ Nursing. Professor of the School of Nursing. University of Costa Rica. Costa Rica. E-mail: ccuberoalpzar@gmail.com

${ }^{3}$ Nursing. San Rafael de Alajuela Hospital. Caja Costarricense del Seguro Social. Costa Rica. E-mail: luzf7494@gmail.com

${ }^{4}$ Nursing. San Rafael de Alajuela Hospital. Caja Costarricense del Seguro Social. Costa Rica. E-mail: jeyronurse04@hotmail.com
} 


\title{
Melhores práticas para a lavagem e manuseio do saco alimentação enteral Kangaroo ${ }^{1}$
}

Instituição: Hospital San Rafael de Alajuela

\author{
Consuelo Cubero Alpízar ${ }^{2}$ \\ Luz Marina Flores Martínez ${ }^{3}$ \\ Jenny Rodríguez Rodríguez ${ }^{4}$
}

\section{CITAÇÃO}

Cubero-Alpízar, C., Flores, L.M. y Rodríguez, J. (2016). Melhores práticas para a lavagem e manuseio do saco alimentação enteral Kangaroo. Rev. Enfermería Actual en Costa Rica, 31, 1-10. DOI: http://dx.doi.org/10.15517/revenf.v0i31.25386

\section{RESUMO}

Introdução. Enteral nutrition é uma ferramenta valiosa na terapia de hospitalizadas, especialmente em unidades de cuidados intensivos, e os pacientes para suas doenças perdem a sua capacidade de ingerir alimentos pacientes.

Método. A metodologia apontando prática clínica baseada em evidências (PCBE) lidar com o estudo e análise de pesquisa primária e original de elevada qualidade científica, localização de resultados que respondam à questão clínica proposta foi aplicada.

Resultado. Nenhum estudo responder diretamente a questão, no entanto, várias investigações fazem alusão ao fato de que lavar as mãos e usar luvas limpas como uma estratégia para a manipulação e lavagem deste dispositivo. A qualidade dos estudos encontrados é baixa, porque eles vêm de uma pesquisa com base em opinião de especialistas e revisão da literatura de baixo qualidade.

Conclusão. Nenhuma evidência significativa para apoiar o uso da técnica médica e cirúrgica asséptica para manusear e lavar o saco de alimentação Canguru Enteral foi encontrado.

Palavras-chave: enteral-nutrição, practice-clinic, wash-de-bag-canguru.

\footnotetext{
${ }^{1}$ Data de recepção: 26 de outubro de 2015

Data de aceitação: 15 de fevereiro de 2016

${ }^{2}$ Enfermeira. Docente de la Escuela de Enfermería. Universidad de Costa Rica. Costa Rica. Correio eletrônico: ccuberoalpzar@gmail.com

${ }^{3}$ Enfermeira. Hospital San Rafael de Alajuela. Caja Costarricense del Seguro Social. Costa Rica. Correio eletrônico: luzf7494@gmail.com

${ }^{4}$ Enfermeira. Hospital San Rafael de Alajuela. Caja Costarricense del Seguro Social. Costa Rica. Correio eletrônico: jeyronurse04@hotmail.com
} 


\section{Revista Electrónica Enfermeria Actual en costa Rica}

\section{INTRODUCCIÓN}

El avance en las tecnologías en salud ha mejorado las expectativas y la calidad de vida de las personas de lo cual nutrición enteral es un ejemplo, debido a que se ha convertido en una valiosa herramienta en la terapia de los pacientes hospitalizados, especialmente en las unidades de cuidado intensivo y en pacientes que por sus patologías pierden la habilidad de ingerir alimentos. Respecto del concepto, la nutrición enteral (NE) es definida como "una forma de nutrición que se aplica a través del tracto gastrointestinal (GI) distal a la cavidad oral, utilizando para ello una sonda, un catéter o un estoma" (Yantis y Velander, 2012, s.p.); es decir, mediante este tipo de alimentación se proporciona soporte nutricional a los pacientes con un tracto gastrointestinal funcional que son incapaces de satisfacer sus necesidades alimenticias por ingestión bucal, ya sea por cirugía de boca, pérdida de conciencia, anorexia u obstrucción esofágica, entre otros (Bonet, 2005).

En relación con lo anterior, Klaassen (2002) destaca las bondades de esta intervención al mencionar que actualmente- se constituye en la técnica más utilizada y recomendada en la asistencia nutricional intensiva, apoyado en el conocimiento reciente de las grandes ventajas fisiológicas que posee, su menor costo y el desarrollo de nuevas fórmulas enterales, así como también de nuevos accesos endoscópicos al tubo digestivo. De igual forma, se han documentado las ventajas del inicio temprano de esta terapia, especialmente en los pacientes de cuidado intensivo, puesto que se disminuye la desnutrición y favorece la disminución en el riesgo de sepsis (Bengmark y Ortiz, 2004).

Entre otros trabajos, se encuentra la investigación de Toussaint, Van Gossum, Ballarin y Arvanitakis (2014) destacan que la nutrición enteral presenta ventajas en contraposición a la nutrición parenteral, ya que es una técnica para la nutrición artificial en pacientes con un accesible y funcional aparato digestivo, ya que se asocia con mejores resultados clínicos y una menor incidencia de sepsis. No obstante, a pesar de todos los beneficios que presenta la NE, no debe dejarse de lado las posibles y a veces graves complicaciones derivadas de su empleo que surgen "en distintas categorías: mecánicas, gastrointestinales, infecciosas, metabólicas y otras" (Sánchez y López, 2014, p.118).

También, una de las principales complicaciones de la NE es la contaminación de las fórmulas empleadas, lo que se puede traducir en diarrea como la más frecuente, sustentado en los datos de Anderson citado por Klaassen (2002), quien establece una asociación significativa entre diarrea y fórmulas enterales que contenían recuentos bacterianos mayores altos. Respecto del tema, para Navajas (2002) hay un vínculo entre contaminación de las fórmulas y los casos de diarrea, el cual tiene serias implicaciones especialmente en aquellos pacientes más vulnerables, así como en cuanto a que las complicaciones infecciosas también tienen como consecuencia un aumento significativo de la estadía hospitalaria, con el costo asociado que ello implica.

Ante esta panorámica, es importante acotar que la literatura señala varios puntos críticos, desde la preparación hasta la administración, relacionados con la contaminación de la fórmula entre los que sobresale como el punto más crítico- la preparación realizada por los técnicos de nutrición (Borges, Campos, Cardoso, André y Serafini, 2010).

Otro aspecto destacable es que a nivel nacional existe una significativa variabilidad en el manejo que, en algunas instituciones, implica un gasto significativo ya que el personal de enfermería aplica durante el 


\section{Revista Electrónica Enfermeria Actual en costa Rica}

procedimiento la técnica aséptica médica y quirúrgica, bata estéril, gorro y guantes estériles, a lo que hay que sumar el tiempo que se requiere para la aplicación de tales principios.

Dado lo anterior, y con la pretensión de que se estandarice la práctica clínica sustentada en la mejor evidencia científica disponible se plantea la siguiente pregunta clínica: ¿En pacientes con nutrición enteral utilizando la bolsa kangaroo, el lavado de la bolsa utilizando la técnica aséptica medica y quirúrgica comparado con el lavado sin técnica, disminuye la presencia de infecciones en el tracto gastrointestinal?

\section{MATERIALES Y MÉTODOS}

Para el desarrollo de este trabajo se utilizó la metodología de práctica clínica basada en evidencia (PCBE), la cual trata de localizar hallazgos que respondan a la pregunta planteada mediante el estudio y análisis de investigaciones primarias y originales de alta calidad científica (Moran, 2001).

La PCBE implica un proceso de cinco etapas que inicia con el planteamiento de la pregunta clínica bajo la modalidad del acrónimo PICO (este se descompone de la siguiente manera $\mathrm{P}=$ persona o población; I= intervención; $\mathrm{C}=$ comparación y $\mathrm{O}=$ resultado esperado) (Santos, Pimenta y Nobre, 2007); dicha pregunta es la siguiente: ¿En pacientes con nutrición enteral utilizando la bolsa kangaroo, el lavado de la bolsa utilizando la técnica aséptica médica y quirúrgica comparado con el lavado sin técnica, disminuye la presencia de infecciones en el tracto gastrointestinal?

En un segundo momento, se elaboró una búsqueda de información relevante y de calidad para dar respuesta a la pregunta planteada, tanto en Google académico como en PubMed. Se empleó los siguientes descriptores en español los cuales fueron posteriormente traducidos al inglés para la recuperación de resultados: "Enteral nutrition, contamination and enteral nutrition AND Bacterial contamination and enteral feeding system AND Enteral tube feeding".

De la búsqueda en google académico, se obtuvo 21600 documentos y en PubMed, 303, por lo que fue necesario establecer el límite de "año", para eliminar aquellos que tuvieran más de seis años: en PubMed quedaron 40 y en google académico, 11300 documentos. De igual forma, se eliminó los documentos que incluían población pediátrica, por lo tanto, quedaron 68 artículos; sin embargo, del total de documentos recuperados únicamente siete de ellos proporcionaron resultados que respondían en forma parcial a la pregunta planteada.

Las siguientes etapas involucran la síntesis de evidencia y el análisis crítico, en las que se utiliza las plantillas de evaluación de la evidencia de CASPe. Finalmente, para la implementación y evaluación de la evidencia las investigadoras sugieren que estas etapas deben llevarse a cabo -luego de un proceso de divulgación entre el personal de salud interesado- propiamente el personal de la institución de salud para que sean ellos los que lleven a cabo el proceso de evaluación respecto de los cambios que se realicen en la práctica clínica. 


\section{RESULTADOS}

Después de realizar la búsqueda y análisis crítico de la literatura que correspondiera a la pregunta clínica que motivó este trabajo, no se encontró una evidencia contundente que demostrara la eficacia del uso de la técnica aséptica médica y quirúrgica con respecto a no utilizar técnica en el lavado de la bolsa Kangaroo para la administración de nutrición enteral. La evidencia encontrada se enfoca en investigaciones relacionadas con la contaminación de la nutrición enteral, la cual indica que existe riesgo en todas las fases de la cadena de preparación (Borges, et al., 2010).

A continuación en la tabla 1 se presenta el resumen de las investigaciones incluidas en este trabajo.

Tabla 1.

Evaluación de documentos recuperados e incluidos

\begin{tabular}{|c|c|c|c|c|c|c|}
\hline Título & Autor & Año & Tipo de estudio & Intervención & Resultado & $\begin{array}{c}\text { Grado de } \\
\text { Recomendación } \\
\text { (gradación de } \\
\text { Oxford) }\end{array}$ \\
\hline $\begin{array}{lr}\text { Protocolo } & \text { de } \\
\text { actuación } & \text { de } \\
\text { enfermería en el } \\
\text { manejo de la } \\
\text { nutrición enteral }\end{array}$ & $\begin{array}{l}\text { Alba, A., Chumillas, } \\
\text { A., Cárcoles, E., Díaz, } \\
\text { R., Guzmán, E., } \\
\text { Jiménez, L., Murcia, } \\
\text { I., Rodríguez, M., } \\
\text { Saiz, M., Segovia, C. } \\
\text { y Valero, I. }\end{array}$ & 2012 & Protocolo & $\begin{array}{l}\text { Revisión } \\
\text { literatura }\end{array}$ & $\begin{array}{l}\text { En el procedimiento para el } \\
\text { manejo al momento de preparar } \\
\text { y administrar la nutrición } \\
\text { enteral (NE), un adecuado } \\
\text { lavado de manos y la } \\
\text { colocación de guantes no } \\
\text { estériles para la manipulación } \\
\text { de los diferentes dispositivos } \\
\text { son prácticas fundamentales }\end{array}$ & Grado 5 \\
\hline $\begin{array}{l}\text { Molecular } \\
\text { Epidemiology of } \\
\text { Microorganisms } \\
\text { Isolated from } \\
\text { Food Workers and } \\
\text { Enteral Feeding of } \\
\text { Public Hospitals } \\
\end{array}$ & $\begin{array}{l}\text { Borges, L., Campos, } \\
\text { M., Cardoso, J., } \\
\text { Andre, M. \& Serafini, } \\
\text { A. }\end{array}$ & 2010 & Exploratorio & $\begin{array}{lr}\text { Recolección } & \text { de } \\
\text { muestras } & \text { en manos } \\
\text { de } & \text { personal } \\
\text { encargado } & \text { de } \\
\text { manipular } & \text { la } \\
\text { nutrición } & \end{array}$ & $\begin{array}{l}\text { La manipulación por parte de } \\
\text { los trabajadores de la } \\
\text { alimentación, el ambiente y } \\
\text { utensilios donde se prepara las } \\
\text { fórmulas, son factores de riesgo } \\
\text { contaminación de las } \\
\text { nutriciones enterales }\end{array}$ & Grado 4 \\
\hline $\begin{array}{l}\text { Effect of an } \\
\text { infection control } \\
\text { programme on } \\
\text { bacterial } \\
\text { contamination of } \\
\text { enteral feed in } \\
\text { nursing homes }\end{array}$ & $\begin{array}{l}\text { Ho, S., Tse, M. \& } \\
\text { Boost, M. }\end{array}$ & 2012 & $\begin{array}{c}\text { Estudio de } \\
\text { control cuasi- } \\
\text { experimental } \\
\text { pre-test post-test }\end{array}$ & $\begin{array}{l}\text { Se recogen muestras } \\
\text { y se aplica un } \\
\text { programa de control } \\
\text { infecciones en grupo } \\
\text { control y se toman } \\
\text { nuevamente } \\
\text { muestras }\end{array}$ & $\begin{array}{l}\text { Se demostró la relación } \\
\text { estrecha entre la alimentación } \\
\text { contaminada y la mala higiene } \\
\text { de las manos }\end{array}$ & Grado 3 \\
\hline $\begin{array}{l}\text { Protocolo en } \\
\text { Nutrición Enteral. } \\
\text { Unidad San Juan } \\
\text { de Dios, Tudela }\end{array}$ & Sebastián, S. & 2014 & $\begin{array}{l}\text { Revisión de } \\
\text { literatura }\end{array}$ & $\begin{array}{l}\text { Se realiza una } \\
\text { búsqueda re de } \\
\text { literatura basada en } \\
\text { la evidencia sobre la } \\
\text { nutrición enteral y } \\
\text { cuidados } \\
\text { enfermería }\end{array}$ & $\begin{array}{l}\text { Señala en el procedimiento para } \\
\text { la administración y } \\
\text { manipulación de la NE el uso } \\
\text { de una "técnica higiénica", } \\
\text { como requisito suficiente para } \\
\text { evitar la incidencia de } \\
\text { complicaciones }\end{array}$ & Grado 5 \\
\hline
\end{tabular}

No obstante, en la investigación de Ho, Tse y Boost (2012) se concluye que la educación y el lavado de manos juegan un papel muy importante para reducir las complicaciones asociadas a contaminación de este tipo de intervención. De acuerdo con la gradación de Oxford (2009), dicha evidencia se ubica en un tercer nivel de recomendación. 


\section{Revista Electrónica Enfermeria Actual en costa Rica}

Por su parte, en la investigación de Borges, et al. (2010) la manipulación por parte de los trabajadores de la alimentación, el ambiente y utensilios donde se prepara las fórmulas, son factores de riesgo para la contaminación de las nutriciones enterales. Esta evidencia tiene un nivel de recomendación 4 de acuerdo con la escala de gradación de la calidad de Oxford.

Por su parte Alba, et al (2012) refieren que en el procedimiento para el manejo al momento de preparar y administrar la nutrición enteral (NE), un adecuado lavado de manos y la colocación de guantes no estériles para la manipulación de los diferentes dispositivos son prácticas fundamentales. Esta recomendación tiene un nivel 5 según la escala de gradación de la calidad de Oxford.

En esta misma línea, la investigación de Sebastián (2014) señala en el procedimiento para la administración y manipulación de la NE el uso de una "técnica higiénica", como requisito suficiente para evitar la incidencia de complicaciones. Se destaca que esta recomendación tiene una gradación de calidad 5, según la escala CEBM (Centro de Oxford para la Medicina basada en la evidencia - Grados de comprobación).

\section{Consideraciones éticas.}

Respecto de los principios biéticos, en el proceso investigativo se respetó los derechos de autor de las obras revisadas y utilizadas evidenciado en la citación correcta de cada trabajo.

\section{DISCUSIÓN}

La variabilidad en la práctica clínica existe en prácticamente todas las disciplinas que se encargan de la atención en salud y parte de las características del lugar donde el profesional ejerce, por lo que, en ocasiones, obedece al desarrollo de una práctica muy sustentada en la tradición más que en el conocimiento científico y también en las características individuales de las personas. Lo anterior es confirmado por Fernández y Richard (2009) para quienes "la variabilidad puede observarse tanto desde un enfoque poblacional como desde un enfoque individual. Su constatación pone en evidencia problemas de calidad de la atención y una práctica poco basada en la investigación más válida" (p.114).

La aplicación de la práctica basada en evidencia permite estandarizar la práctica sustentada en la mejor evidencia científica disponible lo cual repercute directamente en una mejor calidad en la atención y, en algunos casos, en una disminución en los costos de la misma.

En lo que respecta a la NE, aunque no se encontró evidencia que respondiera a la pregunta planteada, las investigaciones de Borges (2010), Ho (2012), Alba (2012) y Sebastián (2014), dejan claro que la manipulación de estos dispositivos es segura utilizando medidas higiénicas básicas como el lavado de manos y el uso de guantes limpios.

Además la investigación de Klaassen, et al.(2002) señala "Numerosos estudios señalan que la manipulación durante la preparación de las fórmulas en polvo, en los así llamados sistemas abiertos de preparación, sería el principal origen de la contaminación bacteriana" (s.p.) 


\section{Revista Electrónica Enfermeria Actual en costa Rica}

Ante esta panorámica y aunado al hecho del problema tan importante que son las infecciones nosocomiales, así como el costo que estas tienen para los sistemas de salud y la sociedad en general, es la relevancia que tiene la revisión de este tema, de manera que se aclare sustentado en evidencia científica si tienen fundamentación científica el uso de técnica quirúrgica para manipular una bolsa de nutrición enteral tipo Kangaroo, la cual tiene horas o días de estar colgando junto a la cama del paciente.

Por otro lado, en su investigación, Elia y Stratton (2008) mencionan el uso de la NE en hogares de ancianos, además de que gran cantidad de pacientes utilizan este tipo de alimentación en sus casas, la cual es manipulada por familiares con una adecuada educación por parte del personal de enfermería. Ante esta situación, es evidente que el uso de técnica aséptica médica y quirúrgica resulta innecesaria, máxime si no está estrechamente relacionada con aumento en las complicaciones infecciosas por manipulación de esta nutrición.

En la misma línea, se destaca la revisión de Toussaint, Gossum, Ballarin y Arvanitakis (2015), quienes mencionan la diarrea entre las complicaciones que se pueden presentar en este tipo de alimentación, la cual se estima se presenta entre un $15 \%$ y un $18 \%$ de los pacientes en cuidado crítico. Dichos autores señalan que los factores de riesgo relacionados con el paciente son desnutrición grave, infección bacteriana -en la cual se ha cultivado el microorganismo Clostridium difficile- y medicamentos. En esta revisión no se identifica la manipulación de las bolsas o dispositivos como un factor de riesgo para la transmisión de enfermedades como la diarrea.

Después de desarrollar la presente revisión, el principal hallazgo fue concluir que no hay evidencia que asocie el lavado de la bolsa Kangaro utilizando la técnica aséptica médica y quirúrgica comparado con el lavado sin técnica, aspecto que varía mucho en la práctica clínica.

Otro aspecto importante es lo que señala la investigación de Heyland, Stephens, Day y McClave (2011), quienes apuntan que una relación inadecuada en la administración de calorías y proteínas aumenta las complicaciones. Esta investigación destaca como las diarreas pueden estar ocasionadas por esta mala prescripción en calorías y proteínas no por contaminación al momento de la administración de este tipo de sustrato.

Otro aspecto por considerar es que no se encontró fundamento científico que respalde la aplicación de la técnica aséptica médica y quirúrgica en la manipulación de la NE con bolsa Kangaroo, lo cual implica además de un correcto lavado de manos la utilización de gorro, cubre bocas guantes y bata estéril, dato que llama la atención por cuanto este tipo de bolsa permanece colgada al lado del paciente en al menos ocho horas y está expuesta a un sinnúmero de microorganismos que circulan en el ambiente hospitalario.

Finalmente, es fundamental acotar la necesidad de realizar investigaciones primarias respecto de este tema que permitan tomar decisiones informadas y respaldadas en la mejor evidencia científica.

\section{CONCLUSIÓN}

No se encontró investigaciones que respalden el lavado de la bolsa Kangaroo utilizando la técnica aséptica quirúrgica. Más si existe información -considerada de baja calidad- relacionada con el hecho de que un adecuado 


\section{Revista Electrónica Enfermeria Actual en costa Rica}

lavado de manos y el uso de guates limpios (técnica médica) son suficientes medidas para evitar la contaminación de la nutrición enteral a nivel hospitalario.

\section{Conflicto de intereses.}

Las autoras declaran no tener conflicto de interes en la realización y publicación científica de este documento.

\section{REFERENCIAS BIBLIOGRÁFICAS}

Alba, A., Chumillas, A., Córcoles, E., Díaz, R., Guzmán, E., Jiménez, L., Murcia, I., Rodríguez, M., Saiz, M., Segovia, C. y Valero, I. (2012). Protocolo de actuación de enfermería en el manejo de la nutrición enteral. Recuperado

http://www.chospab.es/publicaciones/protocolosEnfermeria/documentos/180d78de8e55e21de62aca480e02d 65e.pdf

Bengmark, S. y Ortiz, J. (2004). Nutrición enteral: pasado y futuro. Nutrición Hospitalaria, 19 (2).

Bonet, A., Grau T. y el Grupo de Trabajo de Metabolismo y Nutrición de la Sociedad Española de Medicina Intensiva Crítica y Unidades Coronarias.(2005) Estudio multicéntrico de incidencia de las complicaciones de la nutrición parenteral total en el paciente grave. Nutr. Hosp., 20 (4).

Borges, L., Campos, M., Cardoso, J., André, M. y Serafini, A. (2010). Molecular Epidemiology of Microorganisms Isolated from Food Workers and Enteral Feeding of Public Hospitals. Journal of Food Science, 75(7)

Centro de Oxford para la Medicina basada en la evidencia (2009). Oxford Centre for Evidence-based Medicine Levels of Evidence (March 2009). Recuperado de http://www.cebm.net/oxford-centre-evidence-basedmedicine-levels-evidence-march-2009/

Elia, M. y Stratton, R. (2008). A cost-utility analysis in patients receiving enteral tube feeding at home and in nursing homes. Clinical Nutrition 27: 416-423

Fernández, J. y Richard, M. (2009). Variabilidad de la práctica clínica. Situación actual y retos para enfermería. Enferm Clin. 20(2):114-118

Heyland, D., Stephens, K., Day, A. \& McClave, S. (2011). The success of enteral nutrition and ICU-acquired infections: A multicenter observational study. Clinical Nutrition 30, 148-155

Ho, S., Tse, M. \& Boost, M. (2012). Effect of an infection control programme on bacterial contamination of enteral feed in nursing homes. Journal of Hospital Infection 82: 49-55. 


\section{Revista Electrónica Enfermeria Actual en costa Rica}

Klaassen, J., García, P., Maíz, A. y Campano, M. (2002). Mecanismos de contaminación de las fórmulas para nutrición

enteral.Rev.chil.infectol, 19 (2) www.scielo.cl/scielo.php?script=sci_arttext...10182002000200001

Moran, L. (2001). Práctica de enfermería basada en evidencias. Revista Mexicana de Enfermería Cardiológica, 9 (1-4): 24-30.

Navajas, M., Chacón, D. J., Solvas, J. y Galvez, R. (1992). Bacterial contamination of enteral feeds as a possible risk of nosocomial infection. J Hosp Infect, 21: 111-20

Sánchez, A. y López, L. (2014). Complicaciones gastrointestinales relacionadas con la nutrición enteral domiciliaria. Rev. Esp Nutr Comunitaria, 20(3):118-121

Santos, C., Pimenta, C. y Nobre, M. (2007). Estrategia PICO para la construcción de la pregunta de investigación y la búsqueda de evidencias. Enfermagem, 15(3)

Sebastián, S. (2014). Protocolo en Nutrición Enteral. Unidad San Juan de Dios, Tudela. Recuperado de http://academica-

e.unavarra.es/xmlui/bitstream/handle/2454/14459/TFM_Silvia\%20Sebasti\%C3\%A1n\%20P\%C3\%A9rez.pd $\underline{\text { f?sequence }=4 \& \text { is Allowed }=\mathrm{y}}$

Toussaint, E., Gossum, A., Ballarin, A. y Arvanitakis, M. (2015). Review: Enteral access in adults. Clinical Nutrition 34: 350-358

Toussaint, E., Van, A., Ballarin, A. y Arvanitakis, M. (2014). Review: Enteral access in adults. Clinical Nutrition, $34,350-358$.

Yantis, M. y Velander, R. (2012). Directrices relativas a la nutrición enteral. Recuperado de http://ac.elscdn.com/S0212538212700031/1-s2.0-S0212538212700031-main.pdf? tid=1b62ce64-cc23-11e5-8b8100000aacb35e\&acdnat=1454688866_9f0f53494ff927a97daca25dd50e7968 